\title{
Fractional variational calculus for non-differentiable functions
}

\author{
Agnieszka B. Malinowska
}

\begin{abstract}
The fractional calculus of variations is a subject under strong research. Different definitions for fractional derivatives and integrals are used, depending on the purpose under study. The fractional operators in this paper are defined in the sense of Jumarie. This allows us to work with functions which are nondifferentiable. We present necessary and sufficient optimality conditions for fractional problems of the calculus of variations with a Lagrangian density depending on the free end-points.
\end{abstract}

\section{Introduction}

The Fractional Calculus (FC) is one of the most interdisciplinary fields of mathematics, with many applications in physics and engineering. The history of FC goes back more than three centuries, when in 1695 the derivative of order $\alpha=1 / 2$ was described by Leibniz. Since then, many different forms of fractional operators were introduced: the Grunwald-Letnikov, Riemann-Liouville, Riesz, and Caputo fractional derivatives [23, 34, 38]), and the more recent notions of [11, 19, 24, 25]. FC is nowadays the realm of physicists and mathematicians, who investigate the usefulness of such non-integer order derivatives and integrals in different areas of physics and mathematics (see, e.g., [10, 18, 23]). It is a successful tool for describing complex quantum field dynamical systems, dissipation, and long-range phenomena that cannot be well illustrated using ordinary differential and integral operators (see, e.g., [15, 18, 24, 36]). Applications of FC are found in classical and quantum mechanics, field theories, variational calculus, and optimal control (see, e.g., [14, 17, 20]).

The calculus of variations is an old branch of optimization theory that has many applications both in physics and geometry. Apart from a few examples known since

Agnieszka B. Malinowska

Faculty of Computer Science, Białystok University of Technology, 15-351 Białystok, Poland, email:abmalinowska@ua.pt 
ancient times such as Queen Dido's problem (reported in The Aeneid by Virgil), the problem of finding optimal curves and surfaces has been posed first by physicists such as Newton, Huygens, and Galileo. Their contemporary mathematicians, starting with the Bernoulli brothers and Leibniz, followed by Euler and Lagrange, invented the calculus of variations of a functional in order to solve those problems. Fractional Calculus of Variations (FCV) unifies the calculus of variations and the fractional calculus, by inserting fractional derivatives into the variational integrals. This occurs naturally in many problems of physics or mechanics, in order to provide more accurate models of physical phenomena. The FCV started in 1996 with the work of [36]. Riewe formulated the problem of the calculus of variations with fractional derivatives and obtained the respective Euler-Lagrange equations, combining both conservative and nonconservative cases. Nowadays the FCV is a subject under strong research. Different definitions for fractional derivatives and integrals are used, depending on the purpose under study. Investigations cover problems depending on Riemann-Liouville fractional derivatives (see, e.g., [6, 15, 16, 32]), the Caputo fractional derivative (see, e.g., [1, 7, 28, 30]), the symmetric fractional derivative (see, e.g., [24]), the Jumarie fractional derivative (see, e.g., [4, 5, 19, 20, 21, 22, 27]), and others [2, 11, 14]. For applications of the fractional calculus of variations we refer the reader to [15, 20, 24, 35]. Although the literature of FCV is already vast, much remains to be done.

In this paper we study problems of FCV which are defined in terms of the Jumarie fractional derivatives and integrals. The Euler-Lagrange equations for such problems with and without constraints were recently shown in [4]. Here we develop further the theory by proving necessary optimality conditions for more general problems of FCV with a Lagrangian that may also depend on the unspecified end-points $y(a), y(b)$. More precisely, the problem under our study: to extremize a functional which is defined in terms of the Jumarie fractional operators and having no constraint on $y(a)$ and/or $y(b)$. The novelty is the dependence of the integrand $L$ on the a priori unknown final values $y(a), y(b)$. The new natural boundary conditions (5)(6) have important implications in economics (see [12] and the references therein).

The paper is organized as follows. Section 2 presents the necessary definitions and concepts of Jumarie's fractional calculus. Our results are formulated, proved, and illustrated through examples in Section 3. Main results of the paper include necessary optimality conditions with the generalized natural boundary conditions (Theorem 1) that become sufficient under appropriate convexity assumptions (Theorem 2). We finish with Section 4 of conclusions.

\section{Fractional Calculus}

For an introduction to the classical fractional calculus we refer the reader to [23, 31, 34, 38]. In this section we briefly review the main notions and results from the recent fractional calculus proposed by Jumarie [19, 20, 21]. 
Definition 1. Let $f:[a, b] \rightarrow \mathbb{R}$ be a continuous function. The Jumarie fractional derivative of $f$ is defined by

$$
f^{(\alpha)}(t):=\frac{1}{\Gamma(-\alpha)} \int_{0}^{t}(t-\tau)^{-\alpha-1}(f(\tau)-f(a)) d \tau, \quad \alpha<0,
$$

where $\Gamma(z)=\int_{0}^{\infty} t^{z-1} e^{-t} d t$. For positive $\alpha$, one will set

$$
f^{(\alpha)}(t)=\left(f^{(\alpha-1)}(t)\right)^{\prime}=\frac{1}{\Gamma(1-\alpha)} \frac{d}{d t} \int_{0}^{t}(t-\tau)^{-\alpha}(f(\tau)-f(a)) d \tau,
$$

for $0<\alpha<1$, and

$$
f^{(\alpha)}(t):=\left(f^{(\alpha-n)}(t)\right)^{(n)}, \quad n \leq \alpha<n+1, \quad n \geq 1 .
$$

The Jumarie fractional derivative has the following property:

- The $\alpha$ th derivative of a constant is zero.

- Assume that $0<\alpha \leq 1$, then the Laplace transform of $f^{(\alpha)}$ is

$$
\mathfrak{L}\left\{f^{(\alpha)}(t)\right\}=s^{\alpha} \mathfrak{L}\{f(t)\}-s^{\alpha-1} f(0) .
$$

- $(g(t) f(t))^{(\alpha)}=g^{(\alpha)}(t) f(t)+g(t) f^{(\alpha)}(t), \quad 0<\alpha<1$.

Example 1. Let $f(t)=t^{\gamma}$. Then $f^{(\alpha)}(x)=\Gamma(\gamma+1) \Gamma^{-1}(\gamma+1-\alpha) t^{\gamma-\alpha}$, where $0<$ $\alpha<1$ and $\gamma>0$.

Example 2. The solution of the fractional differential equation

$$
x^{(\alpha)}(t)=c, \quad x(0)=x_{0}, \quad c=\text { constant },
$$

is

$$
x(t)=\frac{c}{\alpha !} t^{\alpha}+x_{0},
$$

with the notation $\alpha !:=\Gamma(1+\alpha)$.

The integral with respect to $(d t)^{\alpha}$ is defined as the solution of the fractional differential equation

$$
d y=f(x)(d x)^{\alpha}, \quad x \geq 0, \quad y(0)=y_{0}, \quad 0<\alpha \leq 1
$$

which is provided by the following result:

Lemma 1. Let $f(t)$ denote a continuous function. The solution of the equation (1) is defined by the equality

$$
\int_{0}^{t} f(\tau)(d \tau)^{\alpha}=\alpha \int_{0}^{t}(t-\tau)^{\alpha-1} f(\tau) d \tau, \quad 0<\alpha \leq 1
$$

Example 3. Let $f(t)=1$. Then $\int_{0}^{t}(d \tau)^{\alpha}=t^{\alpha}, 0<\alpha \leq 1$. 
Example 4. The solution of the fractional differential equation

$$
x^{(\alpha)}(t)=f(t), \quad x(0)=x_{0}
$$

is

$$
x(t)=x_{0}+\Gamma^{-1}(\alpha) \int_{0}^{t}(t-\tau)^{\alpha-1} f(\tau) d \tau .
$$

One can easily generalize the previous definitions and results for functions with a domain $[a, b]$ :

$$
f^{(\alpha)}(t)=\frac{1}{\Gamma(1-\alpha)} \frac{d}{d t} \int_{a}^{t}(t-\tau)^{-\alpha}(f(\tau)-f(a)) d \tau
$$

and

$$
\int_{a}^{t} f(\tau)(d \tau)^{\alpha}=\alpha \int_{a}^{t}(t-\tau)^{\alpha-1} f(\tau) d \tau
$$

For the discussion to follow, we will need the following formula of integration by parts:

$$
\int_{a}^{b} u^{(\alpha)}(t) v(t)(d t)^{\alpha}=\alpha ![u(t) v(t)]_{a}^{b}-\int_{a}^{b} u(t) v^{(\alpha)}(t)(d t)^{\alpha},
$$

where $\alpha !:=\Gamma(1+\alpha)$.

\section{Main Results}

Let us consider the functional defined by

$$
\mathscr{J}(y)=\int_{a}^{b} L\left(x, y(x), y^{(\alpha)}(x), y(a), y(b)\right)(d x)^{\alpha},
$$

where $L(\cdot, \cdot, \cdot, \cdot, \cdot) \in C^{1}\left([a, b] \times \mathbb{R}^{4} ; \mathbb{R}\right)$ and $x \rightarrow \partial_{3} L(t)$ has continuous $\alpha$-derivative. The fractional problem of the calculus of variations under consideration has the form

$$
\begin{gathered}
\mathscr{J}(y) \longrightarrow \text { extr } \\
\left(y(a)=y_{a}\right), \quad\left(y(b)=y_{b}\right) \\
y(\cdot) \in C^{0} .
\end{gathered}
$$

Using parentheses around the end-point conditions means that the conditions may or may not be present.

Along the work we denote by $\partial_{i} L, i=1, \ldots, 5$, the partial derivative of function $L(\cdot, \cdot, \cdot, \cdot, \cdot)$ with respect to its $i$ th argument.

The following lemma will be needed in the next subsection.

Lemma 2. Let $g$ be a continuous function and assume that 


$$
\int_{a}^{b} g(x) h(x)(d x)^{\alpha}=0
$$

for every continuous function $h$ satisfying $h(a)=h(b)=0$. Then $g \equiv 0$.

Proof. Can be done in a similar way as the proof of the standard fundamental lemma of the calculus of variations (see, e.g., [40]).

\subsection{Necessary Conditions}

Next theorem gives necessary optimality conditions for the problem (3).

Theorem 1. Let y be an extremizer to problem (3). Then, y satisfies the fractional Euler-Lagrange equation

$$
\partial_{2} L\left(x, y(x), y^{(\alpha)}(x), y(a), y(b)\right)=\frac{d^{\alpha}}{d x^{\alpha}} \partial_{3} L\left(x, y(x), y^{(\alpha)}(x), y(a), y(b)\right)
$$

for all $x \in[a, b]$. Moreover, if $y(a)$ is not specified, then

$$
\int_{a}^{b} \partial_{4} L\left(x, y(x), y^{(\alpha)}(x), y(a), y(b)\right)(d x)^{\alpha}=\alpha ! \partial_{3} L\left(a, y(a), y^{(\alpha)}(a), y(a), y(b)\right)
$$

if $y(b)$ is not specified, then

$$
\int_{a}^{b} \partial_{5} L\left(x, y(x), y^{(\alpha)}(x), y(a), y(b)\right)(d x)^{\alpha}=-\alpha ! \partial_{3} L\left(b, y(b), y^{(\alpha)}(b), y(a), y(b)\right)
$$

Proof. Suppose that $y$ is an extremizer of $\mathscr{J}$ and consider the value of $\mathscr{J}$ at a nearby function $\tilde{y}=y+\varepsilon h$, where $\varepsilon \in \mathbb{R}$ is a small parameter and $h$ is an arbitrary continuous function. We do not require $h(a)=0$ or $h(b)=0$ in case $y(a)$ or $y(b)$, respectively, is free (it is possible that both are free). Let $j(\varepsilon)=\mathscr{J}(y+\varepsilon h)$. Then a necessary condition for $y$ to be an extremizer is given by $j^{\prime}(0)=0$. Hence,

$$
\int_{a}^{b}\left[\partial_{2} L(\cdot) h(x)+\partial_{3} L(\cdot) h^{(\alpha)}(x)+\partial_{4} L(\cdot) h(a)+\partial_{5} L(\cdot) h(b)\right](d x)^{\alpha}=0
$$

where $(\cdot)=\left(x, y(x), y^{(\alpha)}(x), y(a), y(b)\right)$. Using integration by parts (2) to the second term we get

$$
\begin{aligned}
\int_{a}^{b}\left[\partial_{2} L(\cdot)-\frac{d^{\alpha}}{d x^{\alpha}} \partial_{3} L(\cdot)\right](d x)^{\alpha} & +\left.\alpha ! \partial_{3} L(\cdot)\right|_{x=b} h(b)-\left.\alpha ! \partial_{3} L(\cdot)\right|_{x=a} h(a) \\
& +\int_{a}^{b}\left[\partial_{4} L(\cdot) h(a)+\partial_{5} L(\cdot) h(b)\right](d x)^{\alpha}=0 .
\end{aligned}
$$


We first consider functions $h$ such that $h(a)=h(b)=0$. Then, by the Lemma2 we deduce that

$$
\partial_{2} L(\cdot)=\frac{d^{\alpha}}{d x^{\alpha}} \partial_{3} L(\cdot)
$$

for all $x \in[a, b]$. Therefore, in order for $y$ to be an extremizer to the problem (3), $y$ must be a solution of the fractional Euler-Lagrange equation (47). But if $y$ is a solution of (4), the first integral in expression (8) vanishes, and then condition (7) takes the form

$$
h(b)\left[\int_{a}^{b} \partial_{5} L(\cdot)(d x)^{\alpha}+\left.\alpha ! \partial_{3} L(\cdot)\right|_{x=b}\right]+h(a)\left[\int_{a}^{b} \partial_{4} L(\cdot) d x-\left.\alpha ! \partial_{3} L(\cdot)\right|_{x=a}\right]=0 .
$$

If $y(a)=y_{a}$ and $y(b)=y_{b}$ are given in the formulation of problem (3), then the latter equation is trivially satisfied since $h(a)=h(b)=0$. When $y(b)$ is free, then equation (6) holds, when $y(a)$ is free, then (5) holds, since $h(a)$ or $h(b)$ is, respectively, arbitrary.

In the case $L$ does not depend on $y(a)$ and $y(b)$, by Theorem 1 we obtain the following result.

Corollary 1. [4. Theorem 1] Let y be an extremizer to problem

$$
\mathscr{J}(y)=\int_{a}^{b} L\left(x, y(x), y^{(\alpha)}(x)\right)(d x)^{\alpha} \longrightarrow \text { extr. }
$$

Then, y satisfies the fractional Euler-Lagrange equation

$$
\partial_{2} L\left(x, y(x), y^{(\alpha)}(x)\right)=\frac{d^{\alpha}}{d x^{\alpha}} \partial_{3} L\left(x, y(x), y^{(\alpha)}(x)\right)
$$

for all $x \in[a, b]$. Moreover, if $y(a)$ is not specified, then

$$
\partial_{3} L\left(a, y(a), y^{(\alpha)}(a)\right)=0
$$

if $y(b)$ is not specified, then

$$
\partial_{3} L\left(b, y(b), y^{(\alpha)}(b)\right)=0
$$

Observe that if $\alpha$ goes to 1 , then the operators $\frac{d^{\alpha}}{d x^{\alpha}},(d x)^{\alpha}$ could be replaced with $\frac{d}{d x}$ and $d x$. Thus, in this case we obtain the corresponding result in the classical context of the calculus of variations (see [29, Corollary 1], [12, Theorem 2.1]).

Corollary 2. 29. Corollary 1] If y is a local extremizer for

$$
\begin{gathered}
\mathscr{J}(y)=\int_{a}^{b} L\left(x, y(x), y^{\prime}(x), y(a), y(b)\right) d x \longrightarrow \text { extr } \\
\left(y(a)=y_{a}\right), \quad\left(y(b)=y_{b}\right),
\end{gathered}
$$

then 


$$
\frac{d}{d x} \partial_{3} L\left(x, y(x), y^{\prime}(x), y(a), y(b)\right)=\partial_{2} L\left(x, y(x), y^{\prime}(x), y(a), y(b)\right)
$$

for all $x \in[a, b]$. Moreover, if $y(a)$ is free, then

$$
\partial_{3} L\left(a, y(a), y^{\prime}(a), y(a), y(b)\right)=\int_{a}^{b} \partial_{5} L\left(x, y(x), y^{\prime}(x), y(a), y(b)\right) d x
$$

and if $y(b)$ is free, then

$$
\partial_{3} L\left(b, y(b), y^{\prime}(b), y(a), y(b)\right)=-\int_{a}^{b} \partial_{6} L\left(x, y(x), y^{\prime}(x), y(a), y(b)\right) d x .
$$

\subsection{Sufficient Conditions}

In this section we prove sufficient conditions for optimality. Similarly to what happens in the classical calculus of variations, some conditions of convexity (concavity) are in order.

Definition 2. Given a function $L$, we say that $L(\underline{x}, y, z, t, u)$ is jointly convex (concave) in $(y, z, t, u)$, if $\partial_{i} L, i=2, \ldots, 5$, exist and are continuous and verify the following condition:

$$
\begin{aligned}
L\left(x, y+y_{1}, z+z_{1}, t+t_{1}, u+u_{1}\right)-L(x, y, z, t, u) \\
\geq(\leq) \partial_{2} L(\cdot) y_{1}+\partial_{3} L(\cdot) z_{1}+\partial_{4} L(\cdot) t_{1}+\partial_{5} L(\cdot) u_{1},
\end{aligned}
$$

where $(\cdot)=(x, y, z, t, u)$, for all $(x, y, z, t, u),\left(x, y+y_{1}, z+z_{1}, t+t_{1}, u+u_{1}\right) \in[a, b] \times$ $\mathbb{R}^{4}$.

Theorem 2. Let $L(\underline{x}, y, z, t, u)$ be a jointly convex (concave) in $(y, z, t, u)$. If $y_{0}$ satisfies conditions (4)-(6), then $y_{0}$ is a global minimizer (maximizer) to problem (3).

Proof. We shall give the proof for the convex case. Since $L$ is jointly convex in $(y, z, t, u)$ for any continuous function $y_{0}+h$, we have

$$
\begin{aligned}
\mathscr{J}\left(y_{0}+h\right) & -\mathscr{J}\left(y_{0}\right)=\int_{a}^{b}\left[L \left(x, y_{0}(x)+h(x), y_{0}^{(\alpha)}(x)+h^{(\alpha)}(x), y_{0}(a)+h(a),\right.\right. \\
& \left.\left.y_{0}(b)+h(b)\right)-L\left(x, y_{0}(x), y_{0}^{(\alpha)}(x), y_{0}(a), y_{0}(b)\right)\right](d x)^{\alpha} \\
& \geq \int_{a}^{b}\left[\partial_{2} L(\cdot) h(x)+\partial_{3} L(\cdot) h^{(\alpha)}(x)+\partial_{4} L(\cdot) h(a)+\partial_{5} L(\cdot) h(b)\right](d x)^{\alpha}
\end{aligned}
$$

where $(\cdot)=\left(x, y_{0}(x), y_{0}^{(\alpha)}(x), y_{0}(a), y_{0}(b)\right)$. We can now proceed analogously to the proof of Theorem 1 As the result we get 


$$
\begin{gathered}
\mathscr{J}\left(y_{0}+h\right)-\mathscr{J}\left(y_{0}\right) \geq \int_{a}^{b}\left[\partial_{2} L(\cdot)-\frac{d^{\alpha}}{d x^{\alpha}} \partial_{3} L(\cdot)\right](d x)^{\alpha} \\
h(b)\left[\int_{a}^{b} \partial_{5} L(\cdot)(d x)^{\alpha}+\left.\alpha ! \partial_{3} L(\cdot)\right|_{x=b}\right]+h(a)\left[\int_{a}^{b} \partial_{4} L(\cdot) d x-\left.\alpha ! \partial_{3} L(\cdot)\right|_{x=a}\right]=0,
\end{gathered}
$$

since $y_{0}$ satisfies conditions (4)-(6). Therefore, we obtain $\mathscr{J}\left(y_{0}+h\right) \geq \mathscr{J}\left(y_{0}\right)$.

\subsection{Examples}

We shall provide examples in order to illustrate our main results.

Example 5. Consider the following problem

$$
\begin{aligned}
\mathscr{J}(y)=\int_{0}^{1}\left\{\left[\frac{x^{\alpha}}{\Gamma(\alpha+1)}\left(y^{(\alpha)}\right)^{2}-2 x^{\alpha} y^{(\alpha)}\right]^{2}\right. \\
\left.+(y(0)-1)^{2}+(y(1)-2)^{2}\right\}(d x)^{\alpha} \longrightarrow \text { extr. }
\end{aligned}
$$

The Euler-Lagrange equation associated to this problem is

$$
\frac{d^{\alpha}}{d x^{\alpha}}\left(2\left[\frac{x^{\alpha}}{\Gamma(\alpha+1)}\left(y^{(\alpha)}\right)^{2}-2 x^{\alpha} y^{(\alpha)}\right] \cdot\left[\frac{2 x^{\alpha}}{\Gamma(\alpha+1)} y^{(\alpha)}-2 x^{\alpha}\right]\right)=0 .
$$

Let $y=x^{\alpha}+b$, where $b \in \mathbb{R}$. Since $y^{(\alpha)}=\Gamma(\alpha+1)$, it follows that $y$ is a solution of (9). In order to determine $b$ we use the generalized natural boundary conditions (5)-(6), which can be written for this problem as

$$
\begin{aligned}
& \int_{0}^{1}(y(0)-1)(d x)^{\alpha}=0, \\
& \int_{0}^{1}(y(1)-2)(d x)^{\alpha}=0 .
\end{aligned}
$$

Hence, $\tilde{y}=x^{\alpha}+1$ is a candidate solution. We remark that the $\tilde{y}$ is not differentiable in $[0,1]$.

Example 6. Consider the following problem

$$
\mathscr{J}(y)=\int_{0}^{1}\left[\left(y^{(\alpha)}(x)\right)^{2}+\gamma y^{2}(0)+\lambda(y(1)-1)^{2}\right](d x)^{\alpha} \longrightarrow \min ,
$$

where $\gamma, \lambda \in \mathbb{R}^{+}$. For this problem, the fractional Euler-Lagrange equation and the generalized natural boundary conditions (see Theorem 1) are given, respectively, as

$$
2 \frac{d^{\alpha}}{d x^{\alpha}} y^{(\alpha)}(x)=0
$$




$$
\begin{gathered}
\int_{0}^{1} \gamma y(0)(d x)^{\alpha}=\alpha ! y^{(\alpha)}(0), \\
\int_{0}^{1} \lambda(y(1)-1)(d x)^{\alpha}=-\alpha ! y^{(\alpha)}(1) .
\end{gathered}
$$

Solving equations (11)-13) we obtain that

$$
\bar{y}(x)=\frac{\gamma \lambda \alpha !}{\gamma \lambda+(\alpha !)^{2}(\lambda+\gamma)} x^{\alpha}+\frac{(\alpha !)^{2} \lambda}{\gamma \lambda+(\alpha !)^{2}(\lambda+\gamma)}
$$

is a candidate for minimizer. Observe that problem (10) satisfies assumptions of Theorem 2 Therefore $\bar{y}$ is a global minimizer to this problem. We note that when $\alpha$ goes to 1 problem (10) tends to

$$
\mathscr{K}(y)=\int_{0}^{1}\left[\left(y^{\prime}(x)\right)^{2}+\gamma y^{2}(0)+\lambda(y(1)-1)^{2}\right] d x \longrightarrow \min .
$$

with the solution

$$
y(x)=\frac{\gamma \lambda}{\gamma \lambda+\lambda+\gamma} x+\frac{\lambda}{\gamma \lambda+\lambda+\gamma} .
$$

\section{Conclusion}

In recent years fractional calculus has played an important role in various fields such as mechanics, electricity, chemistry, biology, economics, modeling, identification, control theory and signal processing (see, e.g., [3, 9, 13, 25, 26, 33, 37]). The fractional operators are non-local, therefore they are suitable for constructing models possessing memory. This gives several possible applications of the FCV, e.g., in describing non-local properties of physical systems in mechanics (see, e.g., [10, 24, 35]) or electrodynamics (see, e.g., [8, 39]). The Jumarie fractional derivative is quite suitable to describe dynamics evolving in a space which exhibit coarsegrained phenomenon. When the point in this space is not infinitely thin but rather a thickness, then it would be better to replace $d x$ by $(d x)^{\alpha}, 0<\alpha<1$, where $\alpha$ characterizes the grade of the phenomenon. The fractal feature of the space is transported on time, and so both space and time are fractal. Thus, the increment of time of the dynamics of the system is not $d x$ but $(d x)^{\alpha}$. In this note we generalize some previous results of the FCV (which are defined in terms of the Jumarie fractional derivatives and integrals) by proving optimality conditions for problems of FCV with a Lagrangian density depending on the free end-points. The advantage of using the Jumarie fractional derivative lies in the fact that this derivative is defined for continuous functions, non-differentiable (see, Example 5). Note that the integrand in problem (3) depends upon the a priori unknown final values $y(a)$ and $y(b)$. The present paper indicates how such problems may be solved. 
Acknowledgements This work is partially supported by the Portuguese Foundation for Science and Technology (FCT) through the Systems and Control Group of the R\&D Unit CIDMA, and partially by BUT Grant S/WI/00/2011. The author is grateful to Delfim F. M. Torres for inspiring discussions and useful comments.

\section{References}

1. Agrawal, O.P.: Fractional variational calculus and the transversality conditions. J. Phys. A, 39, 10375-10384 (2006)

2. Almeida R., Torres, D.F.M.: Calculus of variations with fractional derivatives and fractional integrals. Appl. Math. Lett. 22, 1816-1820 (2009)

3. Almeida R., Torres, D.F.M.: Hölderian variational problems subject to integral constraints. J. Math. Anal. Appl. bf 359, 674-681 (2009)

4. Almeida R., Torres, D.F.M.: Fractional variational calculus for nondifferentiable functions, submitted for publication.

5. Almeida R., Malinowska, A.B., Torres, D.F.M.: A fractional calculus of variations for multiple integrals. Application to vibrating string. J. Math. Phys. 51, 033503 (2010)

6. Atanacković, T.M., Konjik, S., Pilipović, S.: Variational problems with fractional derivatives: Euler-Lagrange equations. J. Phys. A. 41, 095201 (2008)

7. D. Baleanu, Fractional constrained systems and Caputo derivatives. J. Comput. Nonlinear Dynam., 3:199-206, 2008

8. Baleanu, D., Golmankhaneh, A.K., Golmankhaneh, A.K., Baleanu, M.C.: Fractional electromagnetic equations using fractional forms. Int. J. Theor. Phys. 48, 3114-3123 (2009)

9. Baleanu, D., Guvenc, Z.B., Machado, J.A.T.: New Trends in Nanotechnology and Fractional Calculus Applications, Springer Science Business Media (2010)

10. Carpinteri, A., Mainardi, F.: Fractals and fractional calculus in continuum mechanics. Springer, Vienna (1997)

11. Cresson, J.: Fractional embedding of differential operators and Lagrangian systems. J. Math. Phys. 48, 033504 (2007)

12. Cruz, P.A.F., Torres, D.F.M., Zinober, A.S.I.: A non-classical class of variational problems. Int. J. of Mathematical Modelling and Numerical Optimisation 1, 227-236 (2010)

13. Debnath, L.: Recent applications of fractional calculus to science and engineering. Int. J. Math. Math. Sci. 54, 3413-3442 (2003)

14. El-Nabulsi, R.A., Torres, D.F.M.: Necessary optimality conditions for fractional action-like integrals of variational calculus with Riemann-Liouville derivatives of order $(\alpha, \beta)$. Math. Methods Appl. Sci. 30, 1931-1939 (2007)

15. El-Nabulsi, R.A., Torres, D.F.M.: Fractional actionlike variational problems. J. Math. Phys. 49, 053521 (2008)

16. Frederico, G.S.F., Torres, D.F.M.: A formulation of Noether's theorem for fractional problems of the calculus of variations. J. Math. Anal. Appl. 334, 834-846 (2007)

17. Frederico, G.S.F., Torres, D.F.M.: Fractional conservation laws in optimal control theory. Nonlinear Dynam. 53, 215-222 (2008)

18. Hilfer, R.: Applications of fractional calculus in physics. World Sci. Publishing, River Edge, NJ (2000)

19. Jumarie, G.: On the representation of fractional Brownian motion as an integral with respect to $(\mathrm{d} t)^{a}$. Appl. Math. Lett. 18, 739-748 (2005)

20. Jumarie, G.: Fractional Hamilton-Jacobi equation for the optimal control of nonrandom fractional dynamics with fractional cost function. J. Appl. Math. Comput. 23, 215-228 (2007)

21. Jumarie, G.: Table of some basic fractional calculus formulae derived from a modified Riemann-Liouville derivative for non-differentiable functions. Appl. Math. Lett. 22, 378-385 (2009) 
22. Jumarie, G.: Analysis of the equilibrium positions of nonlinear dynamical systems in the presence of coarse-graining disturbance in space, J. Appl. Math. Comput. 32, 329-351 (2010)

23. Kilbas, A.A., Srivastava, H.M., Trujillo, J.J.: Theory and applications of fractional differential equations. Elsevier, Amsterdam (2006)

24. Klimek, M.: Lagrangean and Hamiltonian fractional sequential mechanics. Czechoslovak J. Phys., 52, 1247-1253 (2002)

25. Kolwankar, K.M., Gangal, A.D.: Holder exponents of irregular signals and local fractional derivatives. Pramana J. Phys. 48, 49-68 (1997)

26. Machado, J.A.T., Silva, M.F., Barbosa, R.S., Jesus, I.S., Reis, C.M., Marcos, M.G., Galhano, A.F.: Some applications of fractional calculus in engineering. Mathematical Problems in Engineering (2010) doi:10.1155/2010/639801

27. Malinowska, A.B., Sidi Ammi, M.R., Torres, D.F.M.: Composition functionals in fractional calculus of variations, Commun. Frac. Calc. 1 32-40 (2010)

28. Malinowska, A.B., Torres, D.F.M.: Generalized natural boundary conditions for fractional variational problems in terms of the Caputo derivative. Comput. Math. Appl. 59, 3110-3116 (2010)

29. Malinowska, A.B., Torres, D.F.M.: Natural Boundary Conditions in the Calculus of Variations. Math. Methods Appl. Sci. 33, 1712-1722 (2010)

30. Malinowska, A.B., Torres, D.F.M.: Fractional variational calculus in terms of a combined Caputo derivative. In: Podlubny, I., Vinagre Jara, B. M., Chen, YQ., Feliu Batlle, V., Tejado Balsera, I. (eds.) Proceedings of FDA'10, The 4th IFAC Workshop on Fractional Differentiation and its Applications, Badajoz, Spain, October 18-20, 2010, Article no. FDA10-084, 2010, $6 \mathrm{pp}$.

31. Miller, K.S., Ross, B.: An introduction to the fractional calculus and fractional differential equations. Wiley, New York (1993)

32. Odzijewicz, T., Torres, D.F.M.: Calculus of variations with fractional and classical derivatives. In: Podlubny, I., Vinagre Jara, B. M., Chen, YQ., Feliu Batlle, V., Tejado Balsera, I. (eds.) Proceedings of FDA'10, The 4th IFAC Workshop on Fractional Differentiation and its Applications, Badajoz, Spain, October 18-20, 2010, Article no. FDA10-076, 5 pp.

33. Ortigueira, M.D., Machado, J.A.T.: Fractional calculus applications in signals and systems. Signal Processing 86, 2503-2504 (2006)

34. Podlubny, I., Fractional differential equations. Academic Press, San Diego, CA (1999)

35. Rabei, E.M., Ababneh, B.S.: Hamilton-Jacobi fractional mechanics. J. Math. Anal. Appl. 344, 799-805 (2008)

36. Riewe, F.: Nonconservative Lagrangian and Hamiltonian mechanics. Phys. Rev. E. 53, 18901899 (1996)

37. Ross, B.: Fractional calculus and its applications, Springer, Berlin (1975)

38. Samko, S.G., Kilbas, A.A., Marichev, O.I.: Fractional integrals and derivatives. Translated from the 1987 Russian original, Gordon and Breach, Yverdon (1993)

39. Tarasov, V.E.,: Fractional vector calculus and fractional Maxwell's equations. Ann. Physics 323, 2756-2778 (2008)

40. van Brunt, B.: The calculus of variations. Springer, New York (2004) 